\title{
SCREENING, EARLY DETECTION, AND TREATMENT OF PROSTATE CANCER: A EUROPEAN VIEW*
}

\author{
FRITZ H. SCHRÖDER, M.D.
}

Powerful tests are now available for the detection of prostate cancer, both more frequently and at an earlier and usually more curable stage. In some countries of the world, mainly the United States and some European countries, serum prostate-specific antigen (PSA) and rectal examination-based screening is routinely applied to men at risk. In other countries, particularly in Northern Europe, routine application of screening procedures for prostate cancer is not accepted for a number of reasons: knowledge about the natural history of early lesions suggests that indiscriminate use of these tests will lead to overdiagnosis and overtreatment, information on the effectiveness of treatment from randomized trials is unavailable, and no evidence exists that early diagnosis and treatment will lead to an improvement of disease-related and overall mortality. In this article a number of critical and controversial issues of screening for prostate cancer are reviewed. This includes the risk of prostate cancer to patients, the efficacy and acceptability of the screening tests, the issue of overdiagnosis in relation to the natural history, evidence concerning the effectiveness of treatment, and the chances that early treatment may lead to an improvement of prostate cancer mortality. In relation to these points it is concluded that prostate cancer is a frequent cause of death in men and that at present the possibility of early diagnosis and treatment represents the only possibility of cure. The question whether cure is necessary in every identified case or in identifiable subgroups remains unanswered at this time. Although the individual positive predictive value of the screening tests is low, a combination offers higher positive predictive values that are in the range of $70-80 \%$. With proper streamlining of the screening tests, it may be possible in the future to detect one cancer in 2.5-3.0 biopsies. The most efficient use of the screening tests in the general population still remains to be determined. Estimates related to the amount of overdiagnosis are made. Depending on the definition used, overdiagnosis with one round of screening for prostate cancer is probably in the range two- to threefold. The effectiveness of available treatment modalities compared with delayed management has not been studied in a prospective randomized manner. There is, however, indirect evidence, especially relating to the long-term survival of grade 3 patients and to the long-term normalization of serum PSA values after radical prostatectomy, that at least this treatment is effective. In conclusion, the use of the screening tests cannot be witheld from symptomatic men and those who wish to be examined for the presence of prostate cancer. Application to the general population should depend, however, on the result of a prospective randomized study that shows that early detection and treatment will decrease prostate cancer mortality. UROLOGY 46: 62-70, 1995.

$S^{c}$ creening for disease involves the use of diagnostic testing in the asymptomatic general population. Screening must be differentiated from diagnostic testing in symptomatic patients seeking medical consultation. Both types of diagnostic testing may,

*Work presented in this paper is in part supported by the Dutch Cancer Society (NKB), the Prevention Fund of the Netherlands, and by grant no. 90CV01110, item B 6470,713, and 184, of the Europe Against Cancer program of the European Union.

From the Department of Urology, Erasmus University and Academic Hospital Rotterdam, Rotterdam. The Netherlands

Requests for reprints: Fritz H. Schröder, M.D., Department of Urology, Erasmus University and Academic Hospital Rotterdam, P.O. Box 1738, 3000 DR Rotterdam, The Netherlands however, result in early diagnosis of disease. Wide use of screening in the general population is a controversial issue. The 1964 Declaration of Helsinki (and subsequent revision) discussed the issue of screening and concluded that "concerns for the interest of the subject must always prevail over the interests of science and society." The question of whether even a randomized trial of screening for prostate cancer meets the resulting ethical requirements is still subject to debate. ${ }^{2}$ Thus, there are additional ethical concerns for clinicians whose use of diagnostic testing extends beyond the treatment of symptomatic patients.

Several large studies conducted in the United 
States using serum prostate-specific antigen (PSA) measurement, digital rectal examination (DRE), and transrectal ultrasonography (TRUS) have shown that early detection of prostate cancer is possible. ${ }^{3-7}$ In four of these studies, the men were recruited through advertisements, although none of the studies investigated the effect that that selection method may have had on the results. The studies have, however, clearly shown that up to $75 \%$ of the prostate cancer cases detected may be expected to be histologically confined to the prostate. This compares very favorably with detection rates in routine clinical practice in the United States and elsewhere. A survey by the American College of Surgeons showed that $40.8 \%$ of patients diagnosed with prostate cancer had metastatic or locally extensive disease, which is not curable. ${ }^{8}$ Surprisingly, the interpretation of available data on early detection, natural history, and the potential for cure of prostate cancer has differed according to the geographic location of clinicians. Because of these differences, screening remains controversial. The American Cancer Society (ACS) and the American Urological Association (AUA) share views on screening for prostate cancer, which are summarized in the 1993 recommendations of the ACS as follows':

1 . Men aged $\geq 50$ years should undergo an annual examination to detect prostate cancer.

2. DRE and serum PSA measurement should be performed.

3. Screening of risk groups should start earlier.

4. TRUS should only be used in patients with abnormal DRE and/or PSA.

5. Screening will likely only benefit those men with a life expectancy of $>10$ years.

The ACS concluded that, "while an examination for prostate cancer detects tumors at a more favorable stage, reduction in mortality from screening has not yet been documented."

These recommendations are not shared by the medical community in several European countries, especially the Scandinavian countries, the United Kingdom, the Benelux countries, and France. In Germany a general health check, including DRE to detect prostate cancer, was formally introduced into the healthcare system in 1971. Unfortunately, this program has not resulted in solid data that would allow the assessment of DRE as a screening tool.

European experts have not endorsed the ACS and AUA recommendations because of several controversial issues. There is agreement on the essential criteria identified by Wilson and Jungner ${ }^{10}$ in 1968 that provide a baseline for the use of screening tests in the general population. These criteria have been discussed recently by Schröder and Boyle ${ }^{11}$ in relation to prostate cancer. This article will focus on the following parameters, derived from the prerequisites for screening outlined by Wilson and Jungner, about which experts still differ:

1. The disease must be prevalent and present a risk to the patient.

2. The diagnostic tests must be effective and acceptable.

3. The natural history must be sufficiently understood to avoid overdiagnosis.

4. Effective treatment must be available for potentially curable cases.

5. Early treatment must lead to an improvement of disease-related mortality.

\section{PROSTATE CANCER: A LOW RISK DISEASE?}

Prostate cancer is a highly prevalent disease in men aged $>55$ years. It is the second leading cause of cancer death in men, accounting for $1 \%$ of all male deaths and $10-13 \%$ of all cancer deaths in Western countries. Figure 1 illustrates the relationship between prostate cancer incidence and rate of death from prostate cancer in The Netherlands in 19751976. The incidence is approximately twice as high as the rate of death, and both increase with increasing age. ${ }^{12}$ The fact that only one half of men clinically diagnosed with prostate cancer will die of the disease reflects the high rate of intercurrent deaths in this age group. In 1993, in The Netherlands and most other European countries, the ratio of prostate cancer incidence to death rate remained constant at 2:1. In The Netherlands, 3500 men were diagnosed with prostate cancer and 1700 died of the disease. During the same year in the United States, 165,000 patients were diagnosed with prostate cancer and there were 35,000 deaths resulting from the disease. ${ }^{13}$ The higher ratio of incidence to death rate for prostate cancer in the United States of almost 5:1 is likely a result of the use of screening tests in a higher proportion of the US population. A recent description and analysis of this phenomenon has been reported by Lu-Yao and Greenberg. ${ }^{14}$ The increase in incidence in the United States is paralleled by an increase in the use of radical prostatectomy and radiotherapy to the primary tumor.

Do these tumors represent a risk to their carriers warranting aggressive diagnosis and treatment? Recent published reports suggest that the risk of dying as a result of prostate cancer for those who present with localized lesions may be as low as $13 \%$ in the 10-year period following diagnosis. ${ }^{15,16}$ The patient groups analyzed in these reports, however, had a very low prevalence of poorly differentiated cancer (4\%) and a high average patient age (69-70 years), which obviously impacted on the 10 -year cancerrelated survival data. ${ }^{17}$ In contrast, in a retrospective 


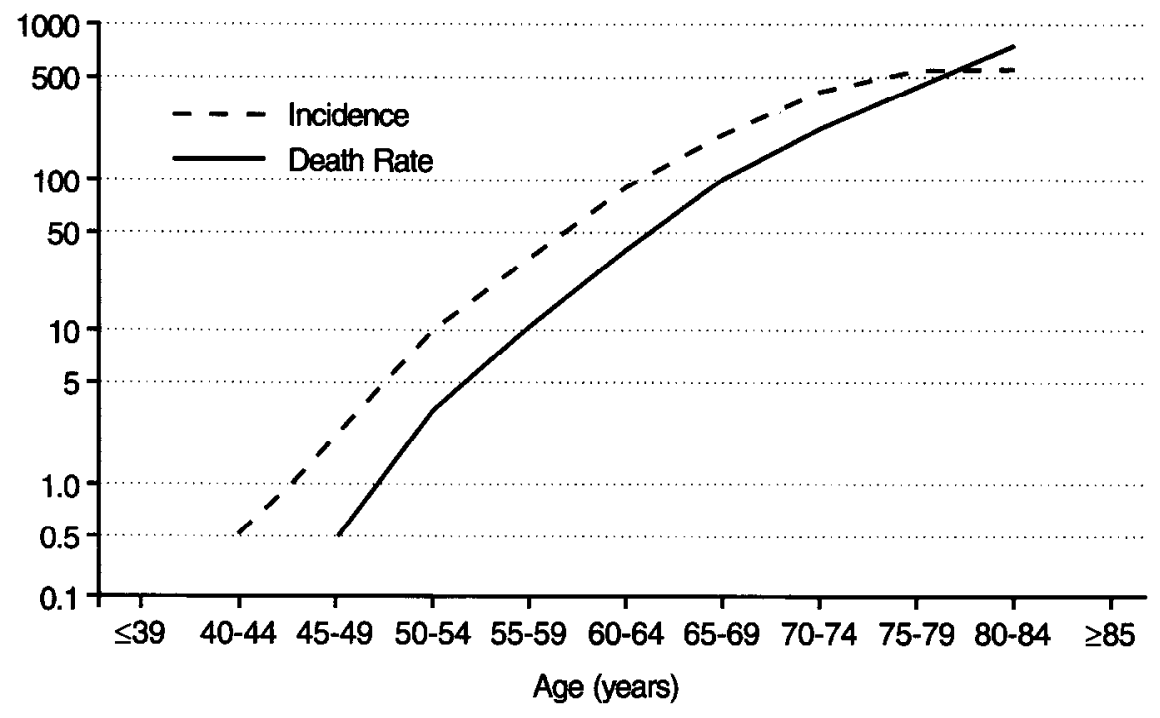

FIGURE 1. Incidence and death rate from prostate cancer by age group in The Netherlands (1975-1976). Data from Dutch Cancer Statistics 1975/76.

study of 536 men from a Swedish cancer registry, Aus ${ }^{17}$ found a 10- and 15-year cancer-related mortality of $50 \%$ and $63 \%$, respectively. As might be expected, he identified a strong relationship between cancer-specific mortality and age that was not apparent in one of the previous studies. ${ }^{16}$

In another cancer registry-based retrospective analysis, Stenman et al. ${ }^{18}$ found a median survival time after diagnosis of 3.6 years. The 10 - and 15 -year survival data were strongly correlated to serum PSA level at the time of diagnosis and, for men with a level $>4.0 \mathrm{ng} / \mathrm{mL}$, the risk of mortality was $48 \%$ and $27 \%$, respectively. The authors concluded that a 10 -year observation period may not be representative because the rate of cancer death at $10-20$ years after diagnosis with prostate cancer remains high. The cancer death rates in the study by Aus were specified for patients with locally confined disease.

Several groups have published decision-analysis models for the diagnosis and management of locally confined prostate cancer. ${ }^{19-21}$ These models rely on the availability of valid natural history data for key assumptions such as metastatic progression rates. All three of the studies cited here used the more favorable natural history data published by Johansson et al. ${ }^{15}$ and thus would be subject to the criticisms already outlined. This points to the need for solid natural history data for prostate cancer. Available data are often confusing and conflicting, requiring interpretation that is dependent on personal biases and individual viewpoints.

Prostate cancer can only be cured if diagnosed in a locally confined state. Routine health care in Western Europe leads to the diagnosis of this disease in ad- vanced stages in $\geq 50 \%$ of cases. Clearly, earlier diagnosis is desirable. Recent case finding studies have enabled histopathologic characterization of tumors found during screening. In a large multicenter study in the United States using serum PSA measurement and DRE, Catalona et al. ${ }^{7}$ reported a rate of organconfined disease of $71 \%$ in 160 men who underwent a radical prostatectomy. Approximately $10 \%$ of these lesions might be considered clinically irrelevant based on tumor volume and grade of differentiation. Epstein et al. ${ }^{22}$ studied 157 patients diagnosed with prostate cancer solely using elevated serum PS $\Lambda$ level (Tlc disease, TNM 1992). Even in this group of patients with nonpalpable and nonvisible disease, the rate of lesions that might be considered clinically insignificant was only $10 \%$. Another $16 \%$ had tumors with a volume of $0.2-0.5 \mathrm{~mL}$ and Gleason grades $<4$. Thus, it appears that whether screening is used in symptomatic patients seeking treatment or in volunteers, advanced screening tests can identify localized and potentially curable disease in about $70-75 \%$ of cases. In addition, morphologic comparison to findings at autopsy and in clinically undiagnosed tumors found in radical cystoprostatectomy specimens can assist clinicians in identifying patients for whom observation is appropriate. ${ }^{23,24}$ However, although these prognostic factors are predictive of favorable outcome, the individual outcome of each tumor remains unknown.

In summary, prostate cancer is a frequent cause of cancer death in men. Although only a very slow increase in prostate cancer mortality is predicted, prostate cancer incidence is rising quickly, especially in the United States. Earlier diagnosis and treatment is 
the only available approach that might be certain to decrease prostate cancer mortality. Whether this decrease in mortality can be achieved through earlier diagnosis and treatment is debated by investigators. Differences in opinion on this one issue are probably at the center of the differing geographic and national views.

\section{DIAGNOSTIC TESTS FOR EARLY DETECTION}

Screening tests should have a sensitivity and specificity of $>90 \%$. If the sensitivity is low, diagnoses will be missed. If the specificity is low, the number of false-positive diagnoses and the number of unnecessary tests performed may be unacceptable. Unfortunately, sensitivity and specificity cannot easily be determined for prostate cancer screening because the actual prevalence of the disease remains unknown. Thus, for prostate cancer screening, positive predictive value (PPV) should define the accuracy of available tests. PPV is expressed as the number of positive tests in patients with the disease divided by the total number of tests performed. The PPV values of DRE, TRUS, and serum PSA level $>4.0 \mathrm{ng} / \mathrm{mL}$ as reviewed by Bentvelsen and Schröder ${ }^{25}$ are shown in Table I and are unacceptably low. The PPV of test combinations has been reviewed by Mettlin et al. ${ }^{26}$ On the basis of data produced in this and similar case finding studies, the ACS and AUA have excluded TRUS as a primary screening test and recommend instead a combination of DRE and serum PSA measurement. The recommendations indicate a biopsy if the serum PSA level is $>4 \mathrm{ng} / \mathrm{mL}$ or the DRE is abnormal.

The use of the three tests in five pilot studies carried out in Rotterdam, The Netherlands, as a part of the European randomized prostate cancer screening study enables a comparison (Table II). These studies differed with respect to the indication for biopsy: two studies indicated biopsy for small lesions discovered at ultrasonography and random biopsies in men with
TABLE I. Positive predictive values (PPVs) of screening tests for prostate cancer

\begin{tabular}{lc}
\hline \multicolumn{1}{c}{ Test } & PPV (\%) \\
\hline Digital rectal examination & 28 \\
Transrectal ultrasonography & 31 \\
Serum prostate-specific antigen & 33 \\
Data from Bentvelsen and Schröder. & \\
\hline
\end{tabular}

nonsuspicious DRE or TRUS and PSA $>4.0 \mathrm{ng} / \mathrm{mL}$. The overall prostate cancer detection rate was $3.5 \%$ (49 cancers in 1402 screened men). Four biopsies were necessary to detect one cancer. It is evident, however, that in the group of men with the PSA between 0 and $2.0 \mathrm{ng} / \mathrm{mL}$, the rate of false-positive findings (i.e., unnecessary biopsy) was extremely high: 76 biopsies ( $37 \%$ of the total number) were necessary in this particular group to detect two prostate cancers. If patients with a PSA between 0.0 and 2.0 $\mathrm{ng} / \mathrm{mL}$ are excluded from the DRE and TRUS groups, the 127 biopsies performed detected 47 cancers. Further analysis showed that with PSA levels $<4.0$ $\mathrm{ng} / \mathrm{mL}$ the proportion of false positive TRUS (24\%) was about two times that of false-positive DRE (13\%). If both tests had been omitted in the group of men with a PSA $<2.0 \mathrm{ng} / \mathrm{mL}$, which amounted to $69.3 \%$ of this total study population, only two prostate cancers would have been missed. Similar data have been reported by Babaian et al. ${ }^{27}$ and Labrie et al. ${ }^{5}$ Although both groups agree that approximately $30 \%$ of all prostate cancers will be found with a PSA $<4.0 \mathrm{ng} / \mathrm{mL}$, rather low proportions of prostate cancers were found in men with a PSA level $<2.0 \mathrm{ng} / \mathrm{mL}$. Babaian et al. ${ }^{27}$ found 10 of 88 prostate cancers (11.4\%) in this group by means of DRE and TRUS. Random biopsies were not taken in this population or by Labrie et al. ${ }^{5}$

These discrepancies illustrate that the exact role of the three available tests in population-based screen-

\section{TABLE II. Number of biopsies performed and prostate cancers detected in relation to serum PSA level in 1402 men screened in five pilot studies in Rotterdam, The Netherlands.}

\begin{tabular}{|c|c|c|c|c|}
\hline \multirow{2}{*}{$\begin{array}{l}\text { PSA Level } \\
(\mathbf{n g} / \mathbf{m L})\end{array}$} & \multirow{2}{*}{$\begin{array}{l}\text { No. Men } \\
(\%)\end{array}$} & \multirow{2}{*}{$\begin{array}{l}\text { No. Biopsies } \\
(\%)\end{array}$} & \multirow{2}{*}{$\begin{array}{c}\text { No. Prostate Cancers } \\
(\%)\end{array}$} & \multirow{2}{*}{$\frac{\text { No. Biopsies }}{\text { No. Prostate Cancers }}$} \\
\hline & & & & \\
\hline $0.0-2.0$ & $971(69)$ & $76(37)$ & $2(4)$ & 38.0 \\
\hline $2.1-4.0$ & $267(19)$ & $39(19)$ & $11(22)$ & 3.5 \\
\hline $4.1-9.9$ & $132(9)$ & $62(31)$ & $21(43)$ & 3.0 \\
\hline$\geq 10.0$ & $32(2)$ & $26(13)$ & $15(31)$ & 1.7 \\
\hline$\overline{\text { Total }}$ & $\overline{1402(100)}$ & $\overline{203(100)}$ & $\overline{49(100)}$ & $\overline{4.1}$ \\
\hline
\end{tabular}

$P S A=$ prostate-specific antigen 
ing is still to be determined. Also, DRE and TRUS are operator-dependent tests. PSA is probably more reliable, but problems of quality control are still to be resolved. The differences seen between the Rotterdam data and Babaian's study may be a result of differing recruitment methods (recruitment from the population registry versus advertisement). The prevalence of prostate cancer in symptomatic populations will likely be higher than that in volunteer populations, as was shown by Cooner et al. ${ }^{28}$ In this urology clinic-based study of symptomatic men, the prostate cancer detection rate was $16 \%$.

Although the use of these tests in large groups of men in the United States seems to "prove" their acceptability, studies of the psychologic impact of the entire early detection procedure, including the screening tests, are unavailable at this time. This information must be determined and made available, since it is relevant to judge the effects of early detection and treatment on quality of life of the screened men. Quality of life evaluations may be decisive in the eventual evaluation of the early detection procedure in general population-based studies.

In summary, individual screening tests for prostate cancer have a low PPV, which increases if combinations of the screening tests are considered. There appears to be some level of serum PSA below which DRE and TRUS should not be applied; the absolute level in the general population has not yet been determined. Determining this level may have great relevance for healthcare policy making, since the use of the more expensive tests may be avoided. Finally, the level of acceptability of the available tests in the general population has not yet been determined. This should preferably be done within the framework of large, randomized, population-based screening studies, since large, nonrandomized, case-finding studies to determine acceptability may be ethically unacceptable.

\section{IS THE AMOUNT OF OVERDIAGNOSIS IN SCREENING FOR PROSTATE CANCER ACCEPTABLE?}

Overdiagnosis of an individual tumor is difficult or impossible to define. There are no prognostic factors that reliably allow pretreatment differentiation of tumors that will become aggressive in the future from those that will not. However, the combination of serum PSA level at the time of diagnosis and tumor volume and grade determined at the time of radical prostatectomy allows a fairly accurate identification of those cases that may become progressive, as shown in the multivariate analysis by Epstein et al. ${ }^{29}$ In this study, "clinically unsignificant" prostate can- cer was defined by a volume of $<0.2 \mathrm{~mL}$; "minimal disease" is characterized as measuring $0.2-0.5 \mathrm{~mL}$, containing no Gleason 4 or 5 pattern, and being confined to the prostate. It is difficult to apply this information to the pretreatment situation, however, because of inaccuracy in determining a grade and tumor volume at the time the diagnosis is made.

Overdiagnosis in a general clinical sense is also difficult to define at this time. It indicates the diagnosis of prostate cancer in men who are not at risk of dying from or having progression of prostate cancer. Overdiagnosis in an epidemiologic sense can be defined as prevalence divided by mortality and with relation to screening efforts as the difference between the screening prevalence divided by mortality and the clinical prevalence divided by mortality:

overdiagnosis $=$ $\frac{\text { screening prevalence }}{\text { mortality }}-\frac{\text { clinical prevalence }}{\text { mortality }}$

Using these formulas for the current situation in Europe as already described, it becomes evident that in most European countries 1 in 2 patients with prostate cancer will die of the disease; in the United States this ratio is reduced to 1 in 5 (the 1993 incidence of prostate cancer in The Netherlands was 3500 and the mortality was 1700 ; in the United States the incidence was 165,000 and mortality was 35,000 ). One of the problems of the definition of overdiagnosis applied is that the mortality changes that may result from ongoing screening efforts are not known. Present prostate cancer mortality may not be the same 10 or 15 years from now. Also, present knowledge about prostate cancer prevalence at screening is limited. The maximal follow-up in case-finding studies amounts to 4 years. Yet figures included in Table III give an impression of the possible extent of overdiagnosis with presently available information. This approach aims to identify the amount of potential overdiagnosis and resulting overtreatment by comparing lifetime prevalence and cross-sectional prevalence obtained at autopsy and calculates the risk of overdiagnosis using prevalence divided by lifetime mortality. It is evident that only about $10 \%$ of those prostate cancers that are detected at autopsy will be detected clinically during a patient's lifetime. That proportion is markedly increased with the application of histologic evaluation to men who have undergone surgical treatment for benign prostatic hyperplasia and in whom previously clinically unsuspected incidental carcinomas are found. Data for lifetime prevalence and lifetime mortality are adapted from the European community data. ${ }^{32}$ The prostate cancer detection 


\begin{tabular}{lcc}
\hline TABLE III. & Risk of overdiagnosis and overtreatment \\
\hline & $\begin{array}{c}\text { Prevalence, Mortality } \\
(\%)\end{array}$ & $\begin{array}{c}\text { Risk of Overdiagnosis and } \\
\text { Overtreatment } \\
\text { (Prevalence/Mortality) }\end{array}$ \\
\hline Autopsy $^{30}$ & 33 & 16.5 \\
Incidental carcinomas $^{31}$ & 12 & 6 \\
Lifetime prevalence $^{32}$ & 4 & 2 \\
Lifetime mortality $^{32}$ & 2 & $(1)$ \\
\hline
\end{tabular}

\begin{tabular}{|c|c|c|}
\hline $\begin{array}{r}\text { TABLE IV. 10-year } \\
T_{1} \text { or } T_{2} \text { prostat } \\
\text { pros }\end{array}$ & $\begin{array}{l}\text { specific survival } \\
\text { r treated by def } \\
\text { my, or external }\end{array}$ & $\begin{array}{l}\text { or patients with Stage } \\
\text { reatment, radical } \\
\text { erapy }\end{array}$ \\
\hline & & ents (\%) \\
\hline & Weighted Mean & 1-Calculated Mortality \\
\hline Deferred treatment* & $229(84)$ & $438(83)$ \\
\hline Radical prostatectomyt & $424(93)$ & $1539(93)$ \\
\hline External radiotherapy & $1035(75)$ & $132(62)$ \\
\hline $\begin{array}{l}* 7.2 \% \text { were grade } 3 \text { tumors. } \\
+16.6 \% \text { were grade } 3 \text { tumors. } \\
\neq 14.0 \% \text { were grade } 3 \text { tumors. }\end{array}$ & & \\
\hline Data from Adolfsson et al. ${ }^{35}$ & & \\
\hline
\end{tabular}

rates in first-round screening have been found to vary between $2.5 \%$ and $5 \%$. The detection rate in annual screening rounds was approximately $0.5 \%$ after 1,2 , and 3 years. ${ }^{33}$ Fortunately, a very large proportion of the cancers found at autopsy remain undetected by presently available screening techniques. The low sensitivity of these tests is desirable in view of the risk of possible resulting overtreatment.

In summary, overdiagnosis with one round of screening for prostate cancer is likely in the range of two- to threefold. Current information seems to indicate that not more than 8-10\% of "pathologist's cancers" are detected. However, the number of patients with nonlethal prostate cancer diagnosed with current definitions of clinically unimportant carcinoma seems to be too small to account for the differences in incidence and mortality seen in the United States at present. ${ }^{22,24}$ The question of how much overdiagnosis is acceptable or necessary can only be answered in the context of the possible decrease in prostate cancer mortality through early diagnosis. Proof of effectiveness of early detection measures is still lacking; it is hoped that the ongoing prospective randomized studies of screening for this disease that use prostate cancer mortality as an endpoint will produce the information necessary to answer this question.

\section{IS PRESENTLY AVAILABLE TREATMENT OF LOCALIZED PROSTATE CANCER EFFECTIVE?}

Unfortunately, no valid randomized studies comparing radical prostatectomy or radiotherapy to delayed treatment regimens have been published. The only randomized study that compares radical prostatectomy plus placebo to placebo treatment only has insufficient power to answer the question. ${ }^{34}$ Furthermore, an ongoing randomized treatment study in Scandinavia is unlikely to produce an answer because of methodologic problems and because the protocol excludes poorly differentiated prostate cancer (grade III). Adolfsson et al. ${ }^{35}$ recently presented a literature overview in which 10-year cancer-specific survival rates in $\mathrm{T} 1$ and $\mathrm{T} 2$ prostate cancer were reevaluated by calculating weighted means and (1 calculated mortality). The review compared the results of deferred treatment to those of radical prostatectomy and external radiotherapy (Table IV). No attempt was made to correct for prognostic factors, although one of the most relevant prognostic factors, the presence of grade III tumors, was identified as being unevenly distributed between the three treatment groups. Assuming that at least half of those patients having grade III tumors may have been cured by surgery, the advantage of radical prostatec- 


\begin{tabular}{|c|c|c|}
\hline \multicolumn{3}{|c|}{$\begin{array}{c}\text { TABLE V. Metastasis-free survival at } 10 \text { years postconservative } \\
\text { treatment versus postradical prostatectomy according to grade of } \\
\text { prostrate tumor originally diagnosed }\end{array}$} \\
\hline & \multicolumn{2}{|c|}{$\begin{array}{l}\text { No. Patients (\%) } \\
\end{array}$} \\
\hline Tumor Grade & Conservative $^{16}$ & $\begin{array}{l}\text { Radical Prostatectomy and } \\
\text { Pelvic Node Dissection }\end{array}$ \\
\hline 1 & $492(81)$ & $156(95)$ \\
\hline 2 & $265(58)$ & $667(83)$ \\
\hline 3 & $62(26)^{*}$ & $123(67) t$ \\
\hline
\end{tabular}

tomy as determined from this overview may increase from $10 \%$ to approximately $15 \%$.

The question of a cure for grade III prostate cancer is a critical point. Until recently, conflicting and limited data have shown that long-term disease-free survival was both not possible ${ }^{36}$ and possible. ${ }^{37}$ Several studies have now reported long-term disease-free survival in patients with poorly differentiated prostate cancer. Table V compares 10 -year metastasis-free survival after conservative treatment with that after radical prostatectomy by grade of malignancy as determined prior to treatment decision. Obviously, historical comparison is not acceptable as proof of the effectiveness of radical prostatectomy. Imbalance of important prognostic factors, such as nodal disease, cannot be corrected for retrospectively. Another unresolvable issue is whether the definitions of grade 3 tumor in the meta-analysis by Chodak et al. ${ }^{16}$ and the report by Zincke et al. ${ }^{38}$ are comparable. Zincke et al. defined Gleason grade $7-10$ as grade III disease, whereas no pathologic review was performed in Chodak's meta-analysis. Also, Zincke et al. applied pelvic node dissection and excluded node-positive patients. Still, the $41 \%$ difference in 10 -year diseasefree survival between the conservative treatment approach and radical prostatectomy is impressive. Recently, an extensive analysis of radical prostatectomies performed at Johns Hopkins Hospital has been presented. $^{39-41}$ Shellhammer ${ }^{41}$ concluded that small grade III tumors do exist and that these lesions are probably curable. In a selected group of 63 patients with prostate cancer graded as Gleason $8-10$, who underwent radical prostatectomy, $44 \%$ had specimen-confined disease, and a $43 \%$ clinical and biochemical progression-free survival rate was observed in this group. ${ }^{39}$

In summary, randomized treatment studies comparing potential curative forms of management with observation are not available. Several randomized treatment studies are underway and it remains to be seen whether these studies will show favorable re- sults. In the meantime, available information suggests that with aggressive treatment a difference in prostate cancer mortality in the range of $15-20 \%$ may emerge. There is increasing evidence that radical prostatectomy is capable of curing poorly differentiated prostate cancer as long as it is organ-confined.

In the meantime, men who present with symptoms or who request screening for prostate cancer cannot be refused the available diagnostic and treatment options. It is essential to provide patients with complete information about the risks of early diagnosis and the risk of overtreatment.

\section{CONCLUSIONS}

The controversies presented in this article are the major reasons for the geographic discrepancies in attitude concerning aggressive screening policies and early treatment of locally confined disease. No real progress can be expected in terms of decreasing cancer mortality in the short-term through improved management of metastatic disease or through the use of prognostic factors to characterize locally confined disease. Despite this, there is still great hesitancy in European countries to accept the US policies on screening and aggressive early management for prostate cancer.

Many European urologists, family physicians, and scientists remain uncertain about the selection of appropriate screening techniques, the risk of uncontrolled overdiagnosis and overtreatment, and the effectiveness of treatment. On the basis of these uncertainties, clinicians in many European countries conclude that screening is premature and that the effectiveness of early detection and early treatment should be studied in randomized screening studies with prostate cancer mortality as a major endpoint. ${ }^{42,43}$ Randomized treatment studies in prostate cancer are difficult to conduct, and this is one reason why results of such studies are not available. A randomized screening study is underway in the United States as part of the National Cancer Institute's com- 
bined screening protocol for prostate cancer, lung cancer, colon cancers, and ovarian cancer. In my view a randomized screening study is the most logical next step. In Europe, a prospective randomized study of screening for prostate cancer is being conducted and coordinated by me.

\section{REFERENCES}

1. Declaration of Helsinki. Recommendations guiding physicians in biomedical research involving human subjects. Adopted by the 18th World Medical Association, Helsinki, Finland, 1964.

2. Adami HO, Baron JA, and Rothman KJ: Ethics of a prostate cancer screening trial. Lancet 343: 958-960, 1994.

3. Catalona WJ, Smith DS, Ratliff TL, Dodds KM, Coplen DE, Yuan JJ, Petros JA, and Andriole GL: Measurement of prostatespecific antigen in serum as a screening test for prostate cancer. N Engl J Med 324: 1156-1161, 1991.

4. Mettlin C, Lee F, Drago J, and Murphy GP: The American Cancer Society National Prostate Cancer Detection Project. Cancer 67: 2949-2958, 1991.

5. Labrie F, Dupont A, Suburu R, Cusan L, Tremblay M, Gomez J-L, and Emond J: Serum prostate specific antigen as pre-screening test for prostate cancer. J Urol 147: 846-852, 1992.

6. Colberg JW, Smith DS, and Catalona WJ: Prevalence and pathological extent of prostate cancer in men with prostate specific antigen levels of 2.9 to $4.0 \mathrm{ng} / \mathrm{mL}$. J Urol 149: 507-509, 1993.

7. Catalona WJ, Richie JP, Ahmann FR, Hudson MA, Scardino PT, Flanigan RC, deKernion JB, Ratliff TL, Kavoussi LR, Dalkin BL, et al: Comparison of digital rectal examination and serum prostate specific antigen in the early detection of prostate cancer: results of a multicenter clinical trial of 6,630 men. J Urol 151: 1283-1290, 1994.

8. Mettlin C, Natarajan N, and Murphy GP: Recent patterns of care of prostate cancer patients in the United States: results from the surveys of the American college of Surgeons Commission on Cancer. Int Adv Surg Oncol 5: 277-321, 1982.

9. Mettlin C, Jones G, Averette H, Gusberg SB, and Murphy GP: Defining and updating the American Cancer Society guidelines for the cancer-related checkup: prostate and endometrial cancers. CA Cancer J Clin 43: 42-46, 1993.

10. Wilson JMG, and Jungner G: Principles and Practice of Screening for Disease. Geneva, WHO, Public Health Paper No. 34,1968 , pp. 1-163.

11. Schröder $\mathrm{FH}$, and Boyle P: Screening for prostate cancernecessity or nonsense? (Editorial) Eur J Cancer 29A: 656-661, 1993.

12. Dutch Cancer Statistics. Morbitity and mortality. 1975/ 1976.

13. Boring CC, Squires TS, and Tong T: Cancer statistics, 1993. CA Cancer J Clin 43: 7-26, 1993.

14. Lu-Yao GL, and Greenberg ER. Changes in prostate cancer incidence and treatment in USA. Lancet 343: 251-254, 1994.

15. Johansson J-E, Adami H-O, Andersson S-O, Bergström R, Holmberg L, and Krusemo UB: High 10-year survival rate in patients with early, untreated prostatic cancer. JAMA 267: 2191-2196, 1992.

16. Chodak GW, Thisted RA, Gerber GS, Johansson JE, Adolfsson J, Jones GW, Chisholm GD, Moskovitz B, Livne PM, and Warner J: Results of conservative management of clinically localized prostate cancer. N Engl J Med 330: 242-248, 1994.
17. Aus G: Prostate cancer: mortality and morbidity after non-curative treatment with aspects on diagnosis and treatment. Scand J Urol Nephrol Suppl 167: 1-41, 1994.

18. Stenman UH, Hakama M, Knekt P, Aromaa A, Teppo L, and Leinonen J: Serum concentrations of prostate specific antigen and its complex with alpha 1-antichymotrypsin before diagnosis of prostate cancer. Lancet 344: 1594-1598, 1994.

19. Fleming C, Wasson JH, Albertsen PC, Barry MJ, and Wennberg JE: A decision analysis of alternative treatment strategies for clinically localized prostate cancer. JAMA 269: 26502658, 1993.

20. Beck JR, Kattan MW, and Miles BJ: A critique of the decision analysis for clinically localized prostate cancer. J Urol 152: 1894-1899, 1994.

21. Krahn MD, Mahoney JE, Eckman MH, Trachtenberg J, Pauker SG, and Detsky AS: Screening for prostate cancer: a decision analytic view. JAMA 272: 779-790, 1994.

22. Epstein JI, Walsh PC, Carmichael M, and Brendler CB: Pathologic and clinical findings to predict tumour extent of nonpalpable (Stage Tlc) prostate cancer. JAMA 271: 368-374, 1994.

23. Ohori M, Wheeler TM, Dunn JK, Stamey TA, and Scardino PT. The pathological features and prognosis of prostate cancers detectable with current diagnostic tests. J Urol 1995 (in press).

24. Stamey TA, Freiha FS, McNeal JE, Redwine EA, Whittemore AS, and Schmid HP: Localized prostate cancer: relationship of tumour volume to clinical significance for treatment of prostate cancer. Cancer 71: 933-938, 1993.

25. Bentvelsen FM, and Schröder FH. Modalities available for screening for prostate cancer. Eur J Cancer 29A: 804-811, 1993.

26. Mettlin C, Lee F, Drago J, and Murphy GP: The American Cancer Society National Prostate Cancer Detection Project. Findings on the detection of early protate cancer in 2,425 men. Cancer 67: 2949-2958, 1991.

27. Babaian RJ, Mettlin C, Kane R, Murphy GP, Lee F, Drago $J R$, and Chesley A: The relationship of prostate-specific antigen to digital rectal examination and transrectal ultrasonography. Findings of the American Cancer Society National Prostate Cancer Detection Project. Cancer 69: 1195-200, 1992.

28. Cooner WH, Mosley BR, Rutherford CJ Jr, Beard JH, Pond HS, Terry WJ, Igel TC, and Kidd DD: Prostate cancer detection in a clinical urological practice by ultrasonography, digital rectal examination and prostate specific antigen. J Urol 143: 1 146-1154, 1990.

29. Epstein JL, Pizov G, and Walsh PC: Correlation of pathologic findings with progression following radical retropubic prostatectomy. Cancer 71: 3582-3593, 1993

30. Franks LM: Proceedings: etiology, epidemiology and pathology of prostatic cancer. Cancer 32: 1092-1095, 1973.

31. Yamabe H, ten Kate FJ, Gallee MP, Schröder FH, Oishi K, Okada $\mathrm{K}$, and Yoshida $\mathrm{O}$ : Stage A prostatic cancer: a comparative study in Japan and The Netherlands. World J Urol 4: 136-140, 1986.

32. Jensen OM; Estève J, Møller H, Renard H: Cancer in the European Community and its members states. Eur J Cancer 26: 1167-1256, 1990.

33. Labrie F, Dupont A, Suburu R, Cusan L, Gomez J-L, Koutsilieris M, Diamond P, Emond J, Lemay M, and Candas B: Optimized strategy for detection of early stage, curable prostate cancer: role of prescreening with prostate specific antigen. Clin Invest Med 16: 426-441, 1994.

34. Graversen PH, Nielsen KT, Gasser TC, Corle DK, and Madsen PO: Radical prostatectomy versus expectant primary treatment in stages I and II prostatic cancer. A fifteen year follow-up. Urology 36: 493-498, 1990. 
35. Adolfsson J, Steineck G, and Whitmore WF: Recent results of management of palpable clinically localized prostate cancer. Cancer 72: 310-322, 1993.

36. Jewett HJ, Bridge RW, Gray GF Jr, and Shelley WM. The palpable nodule of prostatic cancer. Results 15 years after radical excision. JAMA 203: 403-406, 1968.

37. Schröder FH: Prostatic carcinoma: comments on radical surgical treatment. Scand J Urol Nephrol Suppl 55: 181-185, 1980.

38. Zincke H, Bergstralh EJ, Blute ML, Myers RP, Barrett DM, Lieber MM, Martin SK, and Oesterling JE: Radical prostatectomy for clinically localized prostate cancer: long-term results of 1143 patients from a single institution. Clin Oncol 1995 (in press).
39. Partin AW, Lee BR, CarMichael M, Walsh PC, and Epstein Jl: Radical prostatectomy for high grade disease: a reevaluation 1993. J Urol 151: 1583-1586, 1994.

40. Epstein JI, Carmichael MJ, Partin AW, and Walsh PC: Small high grade adenocarcinoma of the prostate in radical prostatectomy specimens performed for nonpalpable disease: pathogenetic and clinical implications. J Urol 151: 1587-1592, 1994.

41. Schellhammer PF. Where treatment may be necessary and possible. J Urol 151: 1593-1594, 1994.

42. Brendler CB: Characteristics of prostate cancer found with early detection regimens. Urology 199546 (suppl 3A): 71-76, 1995.

43. Schroder FH: Prostate cancer: to screen or not to screen? Br Med J 306: 407-408, 1993. 